\title{
Dimensioning BCH Codes for Coherent DQPSK Systems with Laser Phase Noise and Cycle Slips
}

\author{
Miu Yoong Leong, Knud J. Larsen, Gunnar Jacobsen, Sergei Popov, Darko Zibar, and Sergey Sergeyev
}

\begin{abstract}
Forward error correction (FEC) plays a vital role in coherent optical systems employing multi-level modulation. However, much of coding theory assumes that additive white Gaussian noise (AWGN) is dominant, whereas coherent optical systems have significant phase noise (PN) in addition to AWGN. This changes the error statistics and impacts FEC performance. In this paper, we propose a novel semi-analytical method for dimensioning binary Bose-Chaudhuri-Hocquenghem (BCH) codes for systems with PN. Our method involves extracting statistics from pre-FEC bit error rate (BER) simulations. We use these statistics to parameterize a bivariate binomial model that describes the distribution of bit errors. In this way, we relate pre-FEC statistics to post-FEC BER and BCH codes. Our method is applicable to pre-FEC BER around $10^{-3}$ and any post-FEC BER. Using numerical simulations, we evaluate the accuracy of our approach for a target post-FEC BER of $10^{-5}$. Codes dimensioned with our bivariate binomial model meet the target within $0.2 \mathrm{~dB}$ signal-to-noise ratio (SNR).
\end{abstract}

Index Terms-Forward error correction (FEC), BoseChaudhuri-Hocquenghem (BCH) codes, coherent communications, phase noise, cycle slips.

\section{INTRODUCTION}

$\mathbf{C}$ OHERENT optical systems using multi-level modulation have attracted great interest due to their potential to increase capacity. However, such systems are more susceptible to noise and distortions e.g. additive white Gaussian noise (AWGN), laser phase noise (PN), fiber nonlinearities, chromatic dispersion, implementation losses, etc. Depending on the impairment, digital signal processing (DSP) may or may not be suitable to compensate it. For example, chromatic dispersion lends itself well to DSP compensation [1], whereas polarization-dependent gain of Raman amplifiers does not [2], [3]. In the case of laser PN, older analog amplitude shift keying (ASK) systems compensate this by adjusting receiver bandwidth [4], whereas modern coherent systems use DSP. The use of DSP in coherent systems means that a structure

Manuscript received April 22, 2014; revised June 30, 2014; revised July 25, 2014; accepted August 1, 2014.

M.Y. Leong is with Acreo Swedish ICT, Box 1070, 16425 Stockholm, Sweden and KTH Royal Institute of Technology, 16440 Stockholm, Sweden (e-mail: miuyoong.leong@acreo.se).

K.J. Larsen and D. Zibar are with the Technical University of Denmark (DTU), 2800 Kgs. Lyngby, Denmark.

G. Jacobsen is with Acreo Swedish ICT, Box 1070, 16425 Stockholm, Sweden and KTH Royal Institute of Technology, 16440 Stockholm, Sweden.

S. Popov is with KTH Royal Institute of Technology, 16440 Stockholm, Sweden.

S. Sergeyev is with Aston University, Birmingham, B4 7ET, United Kingdom.

Copyright (c) 2013 IEEE. Personal use of this material is permitted. However, permission to use this material for any other purposes must be obtained from the IEEE by sending a request to pubs-permissions@ieee.org. exists, into which forward error correction (FEC) can be easily incorporated.

We consider FEC for coherent systems with laser PN. Traditionally, FEC codes are designed for AWGN channels [5], [6]. In the case of laser PN, various methods for estimating and compensating PN have been investigated [7]-[10]. However, all methods have a non-zero probability of cycle slips. These affect system performance differently, depending on whether or not differential encoding is used. Typically, differential encoding is used when cycle slips occur frequently, and not used when cycle slips occur infrequently.

When differential encoding is not used (e.g. Quadrature Phase Shift Keying (QPSK)), cycle slips give catastrophic burst errors after demodulation. The task is then to mitigate these burst errors. In [11], the authors propose a method to detect the occurrence of cycle slips. Once detected, the direction of the cycle slip $(+\pi / 2$ or $-\pi / 2)$ is determined from the subsequent phase trajectory, and a correction is applied to the affected symbols. In [12], pilot symbols are periodically inserted in order to limit the length of burst errors. A FEC code is then used to correct the errors. Alternatively, instead of performing demodulation and decoding separately, joint coding and modulation can be used. In [13], a coded modulation scheme using low-density parity-check (LDPC) codes is proposed for coherent optical systems with PN.

When differential encoding is used, differential decoding in the receiver corrects the subsequent errors, leaving a single symbol error at the position of each cycle slip. However, the drawback with differential decoding is that each AWGN error gives two symbol errors instead of one. The presence of singleand double-errors changes the error statistics and thereby FEC performance. This is considered for coherent optical systems using LDPC codes in [14]-[16]. In [17], we present semianalytical methods for dimensioning binary Bose-ChaudhuriHocquenghem (BCH) and Reed-Solomon (RS) codes for systems with PN. These codes are well-understood, systematic to construct, and highly predictable. Decoding is straightforward, making them particularly suitable for low-complexity implementations. Furthermore, unlike LDPC codes, BCH codes do not have an error floor. The method for dimensioning binary $\mathrm{BCH}$ codes in [17] assumes that bit errors have a univariate binomial distribution.

In this paper, we extend our previous publication on $\mathrm{BCH}$ codes for PN in two significant ways: 1) we propose a novel method for dimensioning binary $\mathrm{BCH}$ codes based on a bivariate binomial distribution, and 2) we present numerical simulations to compare the accuracy of our bivariate model with the univariate model in [17]. Our approach involves extracting 


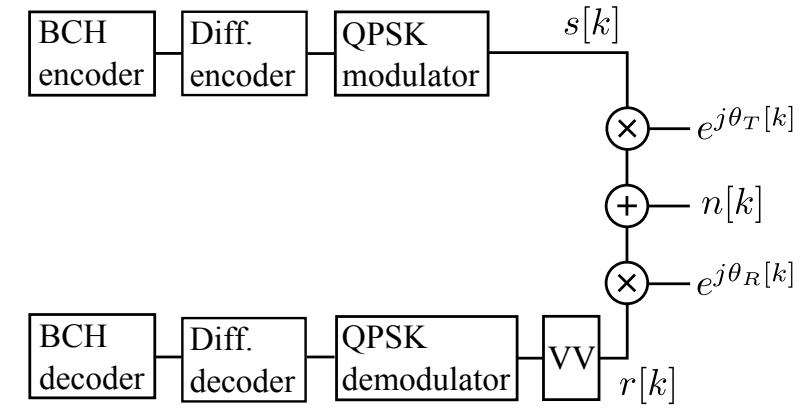

Fig. 1. System model. A random bit sequence is BCH encoded, differentially encoded, and QPSK modulated, yielding signal $s[k]$. Channel impairments are transmitter laser PN $\theta_{T}[k]$, AWGN $n[k]$, and local oscillator (LO) laser PN $\theta_{R}[k]$. Phase estimation on the received signal $r[k]$ is by Viterbi-Viterbi (VV). Finally, the signal is QPSK demodulated, differentially decoded, and $\mathrm{BCH}$ decoded.

parameters from pre-FEC simulations. With these estimates and a given post-FEC bit error rate (BER) target, we determine a suitable code analytically. We aim to correct a pre-FEC BER of around $10^{-3}$. In this paper, we use Differential Quadrature Phase Shift Keying (DQPSK) modulation. Nevertheless, the principles are general and we expect to extend our method to differential $M$-ary Quadrature Amplitude Modulation (QAM) in the future. Ours is a straightforward method, based on a simple model, that enables us to calculate the performance of a low-complexity binary BCH coding scheme for any post-FEC BER with little simulation effort. Compared to our method, the approaches presented in [15], [16] achieve better performance by using soft information. However, those schemes are far more complex to implement, and require extensive simulations for low post-FEC BERs.

This paper is organized as follows: the system model and method for code selection are described in Sec. II. Simulation results and discussion are presented in Sec. III, and the conclusion is in Sec. IV.

\section{System Model And Code Selection}

We consider a single-polarization coherent optical system with baseband-equivalent model shown in Fig. 1. The transmitted signal $s[k]$ is DQPSK modulated, where " 00 " maps to a phase shift of 0 radians, " 01 " to $\pi / 2$, " 11 " to $\pi$, and " 10 " to $-\pi / 2$. The received signal $r[k]$ is

$$
r[k]=s[k] e^{\left(j \theta_{T}[k]+j \theta_{R}[k]\right)}+n[k] \triangleq s[k] e^{j \theta_{N}[k]}+n[k],
$$

where $\theta_{T}[k]$ is transmitter phase noise $(\mathrm{PN}), \theta_{R}[k]$ is local oscillator (LO) $\mathrm{PN}, n[k]$ is AWGN, and $j=\sqrt{-1}$. The total laser $\mathrm{PN} \theta_{N}[k] \triangleq \theta_{T}[k]+\theta_{R}[k]$ is a Wiener process $\theta_{N}[k]=\theta_{N}[k-1]+\Delta \theta_{N}[k]$. The phase changes $\Delta \theta_{N}[k]$ are independent identically distributed (i.i.d.) Gaussian random variables with zero mean and variance $2 \pi \Delta \nu_{N} T_{S}$. The total linewidth $\Delta \nu_{N}=\Delta \nu_{T}+\Delta \nu_{R}$, where $\Delta \nu_{T}$ and $\Delta \nu_{R}$ are the Lorentzian power spectrum full width half maximum (FWHM) linewidths of the transmitter and LO lasers. Symbol time is $T_{S}$ and initial phase $\theta_{N}[0]$ is uniformly distributed $[-\pi, \pi)$.

In the receiver, a commonly-used phase estimation algorithm is Viterbi-Viterbi (VV) [18]. Several variations of the algorithm exist [19], including the use of Wiener filtering [10]

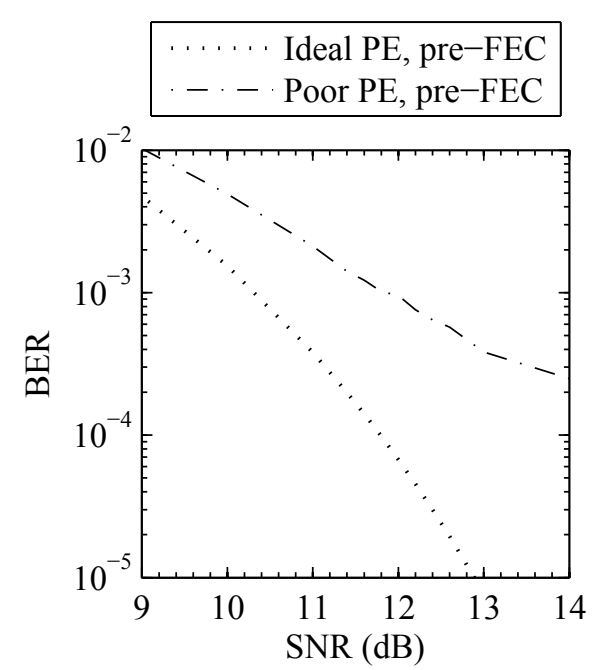

Fig. 2. "Ideal PE pre-FEC" means PN is ideally removed. This is the theoretical BER for coherently demodulated DQPSK with AWGN only. There are no cycle slips; all errors are due to AWGN. "Poor PE pre-FEC" is an example when PN is not ideally removed. Errors are a combination of AWGN and cycle slips. The SNR is for symbols $r[k]$ in Fig. 1.

and the low-complexity Barycenter approximation [20]. In this paper, we choose the original VV [18] as a reasonable starting point for our investigations, and leave detailed comparison of the influence of other VV-based algorithms for future studies. As such, our phase estimate is

$$
\hat{\theta}[k]=\left(\operatorname{unwrap}\left[\arg \left(\sum_{i=k-N}^{k+N} r^{4}[i]\right)\right]\right) / 4 \text {. }
$$

Unwrapping keeps phase jumps within $[-\pi, \pi)$ by adding integer multiples of $2 \pi$. The signal $r[k] \exp (-j \hat{\theta}[k])$ is then coherently demodulated and differentially decoded. The model in Fig. 1 is easily extended to dual-polarization by duplicating all blocks. These operate independently for each polarization except transmitter PN and LO PN, which are the same for both polarizations.

To relate pre- and post-FEC BER to binary $\mathrm{BCH}$ codes, we first use pre-FEC simulations to define an operating range. For a given laser linewidth-symbol time product, $\Delta \nu_{N} T_{S}$, there is an optimum $N$ in (2) that yields performance closest to "ideal phase estimate (PE) pre-FEC" in Fig. 2. However, after optimization, any increase in $\Delta \nu_{N}$ degrades performance. Based on the $\Delta \nu_{N}$ variations that we want to accommodate, we simulate a worst-case "poor PE pre-FEC" curve. For a point on this curve, we record error statistics and dimension codes based on those statistics to achieve a target post-FEC BER.

We record the following error statistics: 1) probability of AWGN error $p_{G}, 2$ ) probability of cycle slips $p_{C}$, and 3 ) correlation coefficient $\rho$. The rest of this paragraph describes how we estimate $p_{G}, p_{C}$, and $\rho$. For a code of block length $n_{B, S}$ bits, we divide the pre-FEC bit error sequence into blocks of that length. For the $m$-th block, we extract the probability of AWGN error $p_{G}[m]$ and probability of cycle slips $p_{C}[m]$ by classifying errors based on their error patterns. For gray-coded DQPSK, an AWGN error of $\pm 90^{\circ}$ gives bit error patterns 
$\{11,101,1001\}$ with probability $\left[\begin{array}{lll}0.25 & 0.5 & 0.25\end{array}\right]$. A cycle slip of $\pm 90^{\circ}$ gives one bit error. The mean probability of the AWGN error is

$$
p_{G}=\frac{1}{M} \sum_{m=1}^{M} p_{G}[m],
$$

where $M$ is the number of blocks used in pre-FEC BER simulations. Similarly, the mean probability of cycle slips is

$$
p_{C}=\frac{1}{M} \sum_{m=1}^{M} p_{C}[m] .
$$

This gives pre-FEC BER $p_{\text {pre }}=2 p_{G}+p_{C}$. The sample correlation coefficient is [21]

$$
\rho=\frac{\sum_{m=1}^{M}\left(p_{G}[m]-p_{G}\right)\left(p_{C}[m]-p_{C}\right)}{\sqrt{\sum_{m=1}^{M}\left(p_{G}[m]-p_{G}\right)^{2} \sum_{m=1}^{M}\left(p_{C}[m]-p_{C}\right)^{2}}} .
$$

To relate these error statistics to binary $\mathrm{BCH}$ codes, we adopt the following model. Let the number of AWGN error patterns that occur in a block be represented by the random variable $Y_{G}$, and the number of cycle slips in a block by the random variable $Y_{C}$. We assume that the occurrence of AWGN error patterns can be modeled as a sequence of Bernoulli trials (biased coin tosses) with probability $p_{G}$. Then $Y_{G}$ is binomial distributed with probability $p_{G}$ and number of trials $n_{B, S}$. Similarly, $Y_{C}$ is binomial distributed $B\left(n_{B, S}, p_{C}\right)$. Furthermore, due to the influence of AWGN on phase estimation ((1)-(2)), $Y_{G}$ and $Y_{C}$ are correlated. The joint probability density function (PDF) of random variables $Y_{G}$ and $Y_{C}$, with correlation $\rho$, and marginal distributions $B\left(n_{B, S}, p_{G}\right)$ and $B\left(n_{B, S}, p_{C}\right)$ is given by [22]

$$
\begin{aligned}
& \operatorname{Pr}\left(Y_{G}=y_{G}, Y_{C}=y_{C}\right) \\
& =\left(\begin{array}{c}
n_{B, S} \\
y_{G}
\end{array}\right) p_{G}^{y_{G}}\left(1-p_{G}\right)^{n_{B, S}-y_{G}} f\left(y_{C} \mid y_{G}\right),
\end{aligned}
$$

where

$$
\begin{aligned}
f\left(y_{C} \mid y_{G}\right)= & \sum_{\left(\gamma_{G}, \gamma_{C}\right) \in \Gamma}\left(\begin{array}{c}
y_{G} \\
\gamma_{G}
\end{array}\right)\left(\begin{array}{c}
n_{B, S}-y_{G} \\
\gamma_{C}
\end{array}\right) \\
& \cdot\left\{p_{C}+\alpha\left(p_{C}-p_{G}\right)+\alpha\right\}^{\gamma_{G}} \\
& \cdot\left\{1-p_{C}-\alpha\left(p_{C}-p_{G}\right)\right\}^{y_{G}-\gamma_{G}} \\
& \cdot\left\{p_{C}+\alpha\left(p_{C}-p_{G}\right)\right\}^{\gamma_{C}} \\
& \cdot\left[\frac{1-p_{C}-\alpha\left(p_{C}-p_{G}\right)+\alpha}{1+\alpha}\right]^{n_{B}, S} \\
& \cdot\left\{1-p_{C}-\alpha\left(p_{C}-p_{G}\right)+\alpha\right\}^{-y_{G}-\gamma_{C}},
\end{aligned}
$$

$\Gamma$ is the set of $\left(\gamma_{G}, \gamma_{C}\right)$ such that

$$
\begin{aligned}
\Gamma= & \left\{\left(\gamma_{G}, \gamma_{C}\right):\right. \\
& \gamma_{G}+\gamma_{C}=y_{C} ; \\
& \gamma_{G}=0,1, \ldots, y_{G} ; \\
& \left.\gamma_{C}=0,1, \ldots, n_{B, S}-y_{G}\right\},
\end{aligned}
$$

and

$$
\alpha=\left[\frac{1}{\rho} \sqrt{\frac{p_{G}\left(1-p_{G}\right)}{p_{C}\left(1-p_{C}\right)}}-1\right]^{-1} .
$$

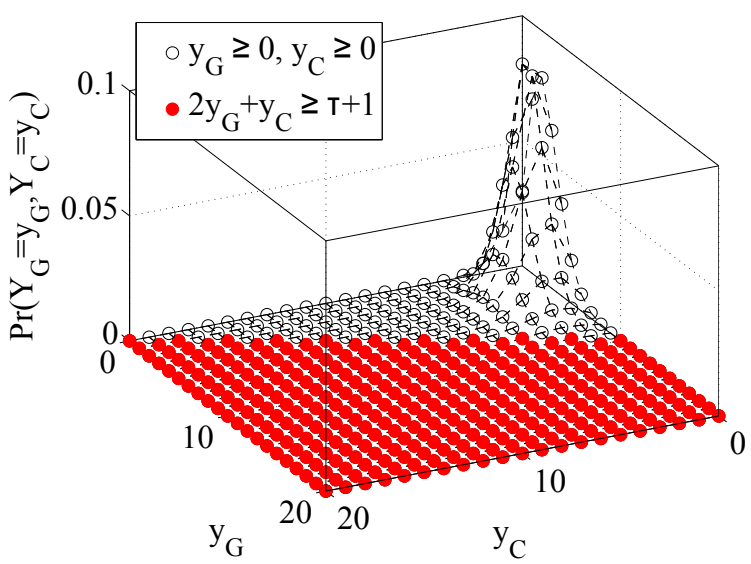

Fig. 3. Joint $\operatorname{PDF} \operatorname{Pr}\left(Y_{G}=y_{G}, Y_{C}=y_{C}\right)$ (6) at "poor PE pre-FEC" circle in Fig. 4. Block length $n_{B, S}=8190$ bits. The volume under the filled balls is the probability of a non-decodable word (10) for a code that can correct $\tau=19$ bit errors.

The joint PDF of $Y_{G}$ and $Y_{C}$ is shown in Fig. 3.

A full-length binary $\mathrm{BCH}$ code $\mathrm{BCH}\left(n_{B}, k_{B}\right)$ corrects $\tau$ bit errors with block length $n_{B}=2^{\mu}-1$ and $n_{B}-k_{B} \leq \mu \tau$ parity-check bits [23]. This is provided that $\mu \geq 3$ and $\tau<2^{\mu-1}$, with $\mu$ being an integer. The code can be shortened by $l_{B}$ bits to $\operatorname{BCH}\left(n_{B, S}, k_{B, S}\right)$, where $n_{B, S}=n_{B}-l_{B}$, $k_{B, S}=k_{B}-l_{B}$, and $n_{B, S}-k_{B, S} \leq \mu \tau$. Full-length codes are a special case of shortened codes where $l_{B}=0$. The decoding algorithm is assumed to be of the boundeddistance type correcting up to $\tau$ errors and leaving the received sequence unchanged in the case of more than $\tau$ errors. We neglect the possibility of decoding to a wrong codeword since this is $(1 / \tau !) \operatorname{Pr}$ (more than $\tau$ errors). The first factor is very small with the relevant $\tau$ (cf. Table I) and it is a good approximation of the ratio between the number of syndromes needed to correct up to $\tau$ errors and the total number of possible syndromes, $2^{n_{B}-k_{B}}$, [24]. Thus, the probability of a non-decodable word is the area under the tail of (6),

$$
P_{B, W}=\sum_{\left(y_{G}, y_{C}: 2 y_{G}+y_{C} \geq \tau+1\right)} \operatorname{Pr}\left(Y_{G}=y_{G}, Y_{C}=y_{C}\right) .
$$

A factor of 2 multiplies $y_{G}$ because AWGN error patterns have 2 bit errors.

Assuming $n_{B, S} p_{\text {pre }} \ll \tau$, we may approximate the probability tail by the terms along the filled/unfilled boundary in Fig. 3. This yields

$$
P_{B, W} \approx \sum_{\left(y_{G}, y_{C}: \tau+3 \geq 2 y_{G}+y_{C} \geq \tau+1\right)} \operatorname{Pr}\left(Y_{G}=y_{G}, Y_{C}=y_{C}\right) .
$$

Three terms are needed to define the boundary in (11) because the bivariate PDF (Fig. 3) is two-dimensional.

Post-FEC BER is

$$
P_{B, p o s t} \approx\left(\frac{\tau+1}{n_{B, S}}\right) P_{B, W} .
$$

Using $p_{G}, p_{C}$, and $\rho$ obtained from pre-FEC simulations and (11)-(12), we calculate the required $\tau$ to meet a target postFEC BER for a chosen block length $n_{B, S}$. The combination $n_{B, S}$ and $\tau$ specifies the $\mathrm{BCH}$ code. 


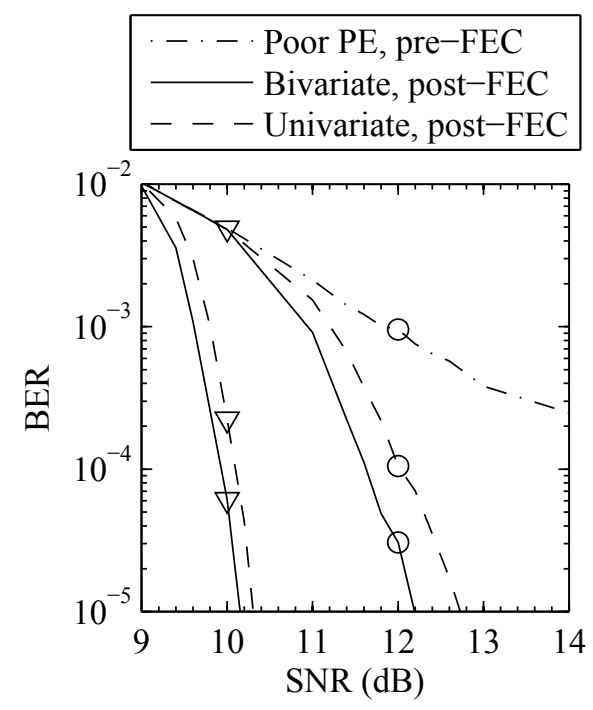

Fig. 4. "Poor PE pre-FEC" is the same as in Fig. 2. We dimension codes for "poor PE pre-FEC" triangle (signal-to-noise ratio (SNR) $10 \mathrm{~dB}$ ) and circle (SNR $12 \mathrm{~dB}$ ). "Bivariate" means codes were dimensioned using the method in Sec. II, and "univariate" means [17]. The codes used for post-FEC simulations are listed in Table I.

\section{RESULTS AND DISCUSSION}

We evaluate our method using Monte-Carlo simulations for the system in Fig. 1. The symbol rate is $1 / T_{S}=28$ Gbaud and $\Delta \nu_{N}<100 \mathrm{kHz}$ works well with VV filter $N=20$ (2). For the limiting case, we simulate "poor PE pre-FEC" in Fig. 4 using laser linewidths $\Delta \nu_{T}=\Delta \nu_{R}=9.8 \mathrm{MHz}$. Pre-FEC BER and error statistics (described in Sec. II for the bivariate model and [17] for the univariate) are calculated using $10^{6}$ bits. Simulations are modeled in VPI [25].

In our example, we aim for a target post-FEC BER of $10^{-5}$ using block length $n_{B, S}=8190$ bits. We dimension codes for two points on the "poor PE pre-FEC" curve, marked with triangle and circle in Fig. 4. For each point, we apply 1) the bivariate model (Sec. II), and 2) the univariate model [17] where bit errors are assumed to be a sequence of i.i.d. Bernoulli trials (biased coin tosses) with bit error probability $p_{p r e}=2 p_{G}+p_{C}$. This yields four different codes, as listed in Table I. Codes for the circle require less overhead than those for the triangle. This is because the circle has lower pre-FEC BER. Codes based on the bivariate model have higher overhead than the univariate model. This is because the univariate model assumes i.i.d. bit errors, whereas the bivariate model includes two correlations: 1) an AWGN error pattern gives two bit errors ( $100 \%$ correlation), and 2) the correlation coefficient $\rho$ (5), which is positive. As such, bit errors in the bivariate model tend to bunch together-blocks with errors have many errors - and thus require stronger codes.

Post-FEC simulations in Fig. 4 use the codes in Table I. Post-FEC BER is calculated using $10^{7}$ post-FEC bits due to simulation limitations. Codes dimensioned using the univariate model have a post-FEC BER of about an order of magnitude worse than target. By contrast, codes dimensioned using the bivariate model have performance well within an order of magnitude of the BER target. This indicates that
TABLE I

CODES USED FOR POST-FEC SIMULATIONS IN FIG. 4. OVERHEAD IS $\left(n_{B, S}-k_{B, S}\right) / k_{B, S}$.

\begin{tabular}{llcc}
\hline Post-FEC curve in Fig. 4 & Code & $\tau$ & Overhead (\%) \\
\hline Circle, univariate & $\mathrm{BCH}(8190,8008)$ & 14 & 2 \\
Circle, bivariate & $\mathrm{BCH}(8190,7943)$ & 19 & 3 \\
Triangle, univariate & $\mathrm{BCH}(8190,7462)$ & 56 & 10 \\
Triangle, bivariate & $\mathrm{BCH}(8190,7345)$ & 66 & 12
\end{tabular}

the bivariate PDF more accurately models the system. The post-FEC bivariate circle is a factor of three higher than the target, and the triangle is a factor of six higher. While this difference is small, it is more than can be attributed to the approximation in (11), and further investigations are needed to understand it. Since a stronger code is used for the triangle, its post-FEC curve is steeper. Both bivariate codes meet the BER target with around $0.2 \mathrm{~dB}$ additional SNR. This would be a negligible difference in practical systems. In other words, using our bivariate model enables us to select binary $\mathrm{BCH}$ codes with minimum overhead that achieve performance close to target.

A system with lower post-FEC BER target would require higher overheads. This is because the volume under the filled balls in Fig. 3 must be smaller and $\tau$ larger. A lower target also improves the accuracy of the leading-order approximation in (11), i.e. approximation error is less at practical post-FEC BERs of $10^{-15}$ than in our example with $10^{-5}$. Additionally, unlike methods that rely solely on simulations to dimension codes, the simulation effort required by our method is modest and does not depend on target post-FEC BER.

As the bivariate model more accurately describes the system, it may also be useful for accurately dimensioning RS codes. A key difference between binary $\mathrm{BCH}$ codes and RS codes is that binary $\mathrm{BCH}$ codes operate on bits whereas RS codes operate on symbols. As such, RS codes are less efficient than binary $\mathrm{BCH}$ codes in correcting single-bit errors, but more efficient for short burst errors. As typical RS codes have symbol sizes of 8 to 12 bits, most AWGN errors affect one symbol, but some affect two symbols. When selecting RS codes, both cases must therefore be considered. Correlation between AWGN errors and cycle slips affects code selection if error bursts are compact in time. The exact expressions for dimensioning RS codes are a topic for future research.

\section{CONCLUSION}

In this paper, we present a semi-analytical method for dimensioning binary $\mathrm{BCH}$ codes. Our method is applicable to differentially-encoded coherent systems impaired by laser PN, cycle slips, and AWGN. Our approach requires only short pre-FEC simulations for parameter estimation. With these estimates and target post-FEC BER, we select codes based on our novel bivariate binomial distribution. Our approach is applicable to pre-FEC BER around $10^{-3}$ and any postFEC BER level. As an example, we consider 28 Gbaud DQPSK and linewidths ranging from $<100 \mathrm{kHz}$ to $19.6 \mathrm{MHz}$. Numerical simulations show that the bivariate model predicts code performance at post-FEC BER $10^{-5}$ to an accuracy of 0.2 
dB SNR. In other words, our method accurately predicts code performance without requiring lengthy post-FEC simulations.

Our approach is novel, general, and easily adapted to different phase estimation algorithms and laser linewidth ranges. It facilitates low-complexity implementations using (cheap) lasers with high PN, even in high-performance coherent systems requiring any low post-FEC BER (e.g. down to $10^{-15}$ and below). Future research includes extending our investigations to interleaving, RS codes, higher-order modulations, and the influence of equalization-enhanced phase noise [26]. Other possibilities include applying the bivariate model to the design of new coding schemes, and evaluating the influence of different Viterbi-Viterbi (VV)-based algorithms e.g. [10], [19], [20].

\section{ACKNOWLEDGMENT}

This work was supported by Vetenskapsrådet (agreement no. 0379801; decision date 2011-10-26), the Engineering and Physical Sciences Research Council (EPSRC) project UNLOC EP/J017582/1, and FP7-PEOPLE-2012-IAPP (project GRIFFON, no. 324391).

\section{REFERENCES}

[1] T. Xu, G. Jacobsen, S. Popov, J. Li, E. Vanin, K. Wang, A. T. Friberg, and Y. Zhang, "Chromatic dispersion compensation in coherent transmission system using digital filters," Opt. Express, vol. 18, no. 15, pp. $16243-$ 16257 , July 2010.

[2] A. Berntson, S. Popov, E. Vanin, G. Jacobsen, and J. Karlsson, "Polarisation dependence and gain tilt of Raman amplifiers for WDM systems," in Proc. OFC, 2001, pp. MI2-1-MI2-3.

[3] S. Popov and E. Vanin, "Polarization dependence of Raman gain on propagation direction of pump and probe signal in optical fibers," in Proc. CLEO, May 2001, pp. 146-147.

[4] I. Garrett and G. Jacobsen, "Phase noise in weakly coherent systems," Optoelectronics, IEE Proceedings J, vol. 136, no. 3, pp. 159-165, June 1989.

[5] F. Chang, K. Onohara, and T. Mizuochi, "Forward error correction for $100 \mathrm{G}$ transport networks," Communications Magazine, IEEE, vol. 48, no. 3, pp. S48-S55, Mar 2010.

[6] A. Leven and L. Schmalen, "Status and recent advances on forward error correction technologies for lightwave systems," in Proc. ECOC, Sept 2013, We.2.C.1

[7] S. Bilal, A. Carena, C. Fludger, and G. Bosco, "Dual stage CPE for 64-QAM optical systems based on a modified QPSK-partitioning algorithm," IEEE Photon. Technol. Lett., vol. 26, no. 3, pp. 267-270, Feb 2014.

[8] A. Meiyappan, H. Kim, and P.-Y. Kam, "A low-complexity, low-cycleslip-probability, format-independent carrier estimator with adaptive filter length," J. Lightw. Technol., vol. 31, no. 23, pp. 3806-3812, Dec 2013.

[9] H. Cheng, Y. Li, F. Zhang, J. Wu, J. Lu, G. Zhang, J. Xu, and J. Lin, "Pilot-symbols-aided cycle slip mitigation for DP-16QAM optical communication systems," Opt. Express, vol. 21, no. 19, pp. 22166 22 172, Sept 2013.

[10] M. Taylor, "Phase estimation methods for optical coherent detection using digital signal processing," J. Lightw. Technol., vol. 27, no. 7, pp. 901-914, Apr 2009.

[11] Y. Gao, A. Lau, C. Lu, Y. Dai, and X. Xu, "Blind cycle-slip detection and correction for coherent communication systems," in Proc. ECOC, Sept 2013, P.3.16.

[12] K. Roberts and A. Khandani, "Parity frame," Patent US 2013/0 191696 A1, July, 2013.

[13] I. Djordjevic and T. Wang, "On the LDPC-coded modulation for ultrahigh-speed optical transport in the presence of phase noise," in Proc. OFC, 2013, OM2B.1.

[14] M. Magarini, L. Barletta, A. Spalvieri, A. Leven, M. Pepe, and G. Gavioli, "Impact of nonideal phase reference on soft decoding of differentially encoded modulation," IEEE Photon. Technol. Lett., vol. 24, no. 23, pp. 2179-2182, Dec 2012.
[15] J. Karaki, R. L. Bidan, and E. Pincemin, "A simple and highperformance method for combining soft-decision FEC with differential encoding in 100 Gbps dual-polarization QPSK system," in Proc. OFC, Mar 2014, M3A.7.

[16] T. Koike-Akino, K. Kojima, D. Millar, K. Parsons, Y. Miyata, W. Matsumoto, T. Sugihara, and T. Mizuochi, "Cycle slip-mitigating turbo demodulation in LDPC-coded coherent optical communications," in Proc. OFC, Mar 2014, M3A.3.

[17] M. Y. Leong, K. J. Larsen, G. Jacobsen, S. Popov, D. Zibar, and S. Sergeyev, "Novel BCH code design for mitigation of phase noise induced cycle slips in DQPSK systems," in Proc. CLEO, June 2014, STu3J.6.

[18] A. Viterbi and A. Viterbi, "Nonlinear estimation of PSK-modulated carrier phase with application to burst digital transmission," IEEE Trans. Inf. Theory, vol. IT-29, no. 4, pp. 543-551, July 1983.

[19] S. Savory, "Digital coherent optical receivers: Algorithms and subsystems," Selected Topics in Quantum Electronics, IEEE Journal of, vol. 16, no. 5, pp. 1164-1179, Sept 2010.

[20] S. Hoffmann, R. Peveling, T. Pfau, O. Adamczyk, R. Eickhoff, and R. Noe, "Multiplier-free real-time phase tracking for coherent qpsk receivers," IEEE Photon. Technol. Lett., vol. 21, no. 3, pp. 137-139, Feb 2009.

[21] C. W. Helstrom, Probability and Stochastic Processes for Engineers. Macmillan, 1984.

[22] A. Biswas and J.-S. Hwang, "A new bivariate binomial distribution," Statistics \& Probability Letters, vol. 60, no. 2, pp. 231-240, 2002.

[23] S. Lin and D. J. Costello, Error Control Coding, 2nd ed. Prentice Hall, 2004.

[24] R. McEliece and L. Swanson, "On the decoder error probability for Reed-Solomon codes (corresp.)," IEEE Trans. Inf. Theory, vol. 32, no. 5, pp. 701-703, Sept 1986.

[25] www.vpiphotonics.com

[26] W. Shieh and K.-P. Ho, "Equalization-enhanced phase noise for coherent-detection systems using electronic digital signal processing," Opt. Express, vol. 16, no. 20, pp. 15 718-15 727, Sept 2008. 